\title{
Four Generations of Quality- GxP pharmaceutical quality assurance, an alternative track?
}

\section{John P. Hammond}

Technical Manager, Starna Scientific Limited, 52-54 Fowler Road, Hainault, Essex IG6 3UT, UK

\section{Introduction}

This article, the fifth in the series details the history and evolution of the GxP Quality Assurance environment, used exclusively in the pharmaceutical and related support industries. In the above pneumonic $x=L$ or $M$, relating the Good Practice to either the "Laboratory" or "Manufacturing", amongst others.

Why "alternative track"?

"...two nations divided by a common language"

The above quotation has been variously attributed to either Oscar Wilde or George Bernard Shaw, and in the context of this article series, one would suggest that this quotation could be rewritten as: "...ISO and GXP, two Quality Assurance systems divided by a common language"

Or perhaps more contentiously as shown in Figure 1? Where, for the sake of argument, the left side track is "GxP" and the right-hand track is "ISO". We will discuss this analogy as we progress through this article.

\section{DOI: $10.1255 /$ sew.2021.a28}

(c) 2021 The Author

Published under a Creative Commons BY-NC-ND licence

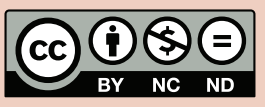

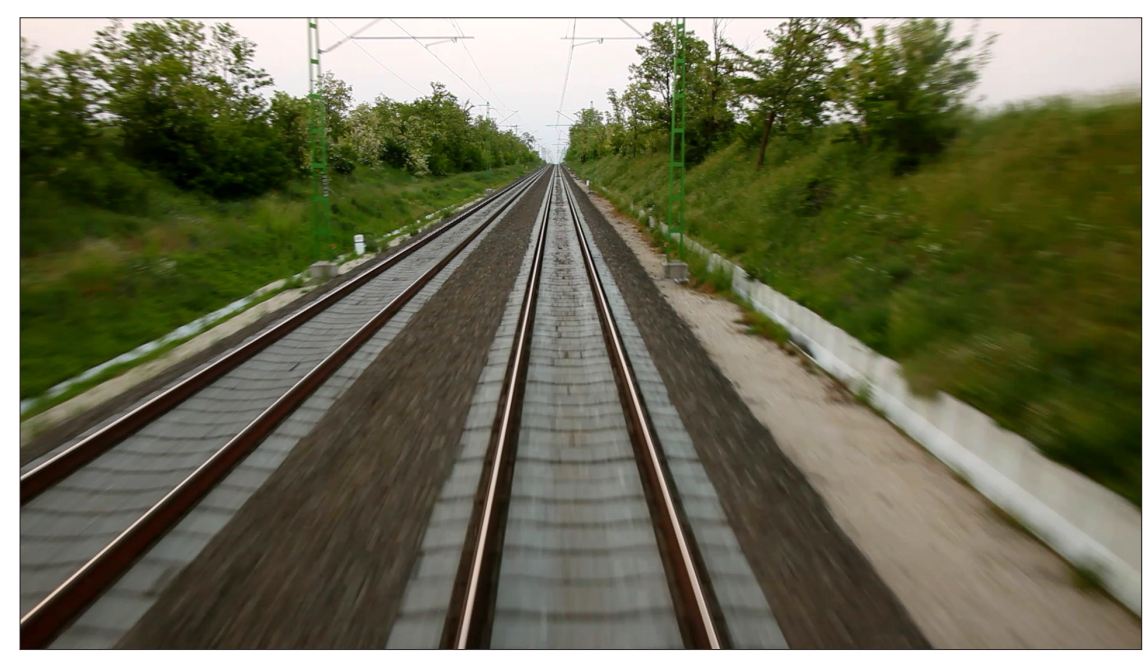

Figure 1. GXP and ISO: on different tracks?

\section{What is GxP?}

GxP are the quality standards and regulations for a specific field or activity. GxP revolves around two main regulatory pillars: accountability and traceability. Accountability refers to the ability to accurately demonstrate that the assigned personal contribution to any process is correctly recorded. Traceability is the process by which a given pathway can be established as an unbroken chain of events. To be compliant, organisations need to document and log every action in the development or production of a product or project. Here we see the first, but not last, commonalities with an ISO 17xxx environment, i.e. the Traceability requirements in an ISO/IEC 17025 accredited process, identification control etc.

\section{What's the purpose of GxP?}

First and foremost, GxP exists to protect us, the consumer of a manufactured product. The guidelines are created and enforced by national or international regulatory agencies, e.g. the European Medicines Agency (EMA) in Europe, the Medicines Control Agency (MCA) in the UK, the Food and Drug Administration (FDA) in the USA etc.; or the global International Council for Harmonization of Technical Requirements for Pharmaceuticals for Human Use ( $\mathrm{ICH})$, to ensure that products, research and projects are done safely and that the end products are safe to use.

The guidelines themselves establish the minimum requirements that an organisation needs to meet to ensure 
that the quality of their goods or services, and thereby the safety, is consistently high and, therefore, are deemed essential for food, pharmaceutical, medical device and life sciences organisations.

Though there are multiple GxPs, the three most usually encountered are described below.

\section{Good Manufacturing Practice [GMP]}

GMP are the guidelines recommended by agencies for the authorisation and control of manufacturing of products such as drugs, medical devices, active pharmaceutical ingredients (APIs) etc. Adhering to these guidelines assure the agencies about the quality of the products and that the manufacturers have taken every possible measure to ensure the safety of the product.

\section{Good Laboratory Practice (GLP)} These are the standards set by the regulatory authority for non-clinical laboratory tests and studies conducted for assessing the safety and efficacy of the product. GLPs are a set of standards which define the framework for a non-clinical study and states how they should be performed, evaluated, reported etc.

\section{Good Clinical Practices (GCP)}

GCP are international quality standards defined by the International Conference on Harmonization $(\mathrm{ICH})$ that state the clinical trial regulations for the products that require testing on human subjects. The standards outline the requirements of a clinical trial and the roles and responsibilities of the officials involved in it. It ensures that no human experiments are performed just for the sake of medical advancement.

\section{Pre-history: the years before 1940}

Unusually in this series, there are significant background events before 1945 in relation to this topic, and these are excellently documented in a highly informative article from 2000, ${ }^{1}$ and an extracted chronological timeline is shown below.

In 1905, a book written by Upton Sinclair, called The Jungle helped catalyse public opinion for change.

As a result of the impact the above book had on the American public, Congress passed the Pure Food and Drug Act in 1906, and for the first time it became illegal to sell contaminated (adulterated) food or meat. Also, for the first time, labelling had to be truthful, i.e. no one could make exorbitant claims on a label anymore.

However, in 1933 an FDA exhibit of dangerous food, medicines, medical devices and cosmetics illustrated the shortcomings of the 1906 law, and when Sulfa drugs were introduced in 1935, one company used diethylene glycol, a poisonous solvent and chemical analogue of antifreeze, in an oral "elixir of sulfanilamide" with the unstated consequences.

In response, Congress passed the Federal Food, Drug and Cosmetic (FD\&C) Act of 1938. For the first time, companies were required to prove that their products were safe before marketing them.

$1^{\text {st }}$ Generation: the years between 1940 and 1975 In 1941, external to World events of the time, 300 people were killed or injured by one company's sulfathiazole tablets, a sulfa drug tainted with the sedative, phenobarbital. This event caused the FDA to revise manufacturing and quality control requirements drastically, leading to what would later be called GMPs. The Public Health Services Act, passed in 1944, covered a broad spectrum of concerns, including regulation of biological products and control of communicable diseases.

During WWII, batch certification became a FDA requirement for certain drugs, insulin in 1941 and penicillin in 1945; later expanded to all antibiotics. However, by 1983, the requirement for batch certification of drugs was dropped.

In the 1960s, Thalidomide was marketed in Europe as a sleeping pill and to treat morning sickness. When regulatory agencies gave permission to sell the drug for that indication, they had no knowledge of its serious side effects. It turned out to be teratogenic: it caused serious deformities in developing foetuses. Children whose mothers took thalidomide in the first trimester were born with severely deformed arms and legs. An estimated 10,000 cases of infant deformities in Europe were linked to Thalidomide use.

Significantly, this product was not allowed on the market in the United

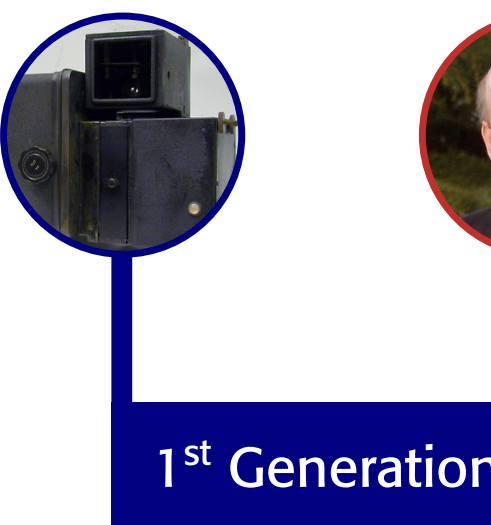

1940

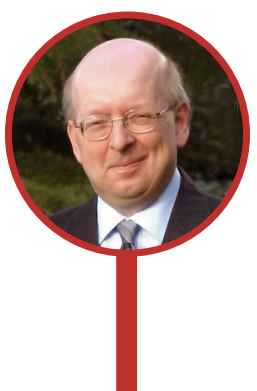

$2^{\text {nd }}$ Generation

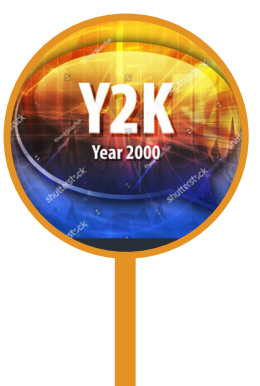

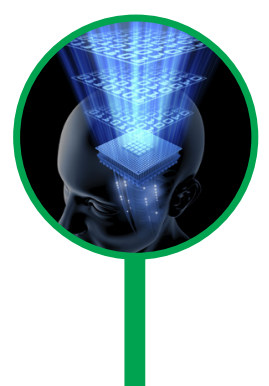

$4^{\text {th }}$ Generation 
States; and, like many other historical tragic events, Thalidomide galvanised public opinion. Two USA legislators, Kefauver and Harris, pushed morestringent legislation through Congress that required companies to test not only to ensure that products were safe, but that they were efficacious for their intended uses. Regulating clinical trials, the amendments required drugs to be tested in animals before people. They made investigators responsible for supervising drugs under study. Manufacturers were expected to inform participants if a drug was being used for investigational purposes and to obtain their consent before testing it on them. Drugs had to be shown to work before going on the market. Manufacturers were required to report unexpected harm (adverse events). And the FDA was given authority to regulate advertising of prescription drugs.

These events in no small part generated the impetus for the changes in the next generation.

\section{$2^{\text {nd }}$ Generation: the years 1975 to 2000}

The 1970s were a turning point for product regulation. In the USA, GMPs for drugs (21 CFR Parts 210 and 211) and medical devices (21 CFR 820) were made final in 1978. They were intended to help ensure the safety and efficacy of all products.

The regulation contained the minimum current good manufacturing practice for methods to be used in, and the facilities or controls to be used for, the manufacture, processing, packing or holding of a drug to assure that such drug meets the requirements of the act as to safety. In addition, the drug must have the identity and strength, and meet the quality and purity characteristics that it purports to be.

GMP requirements for devices were intended "to govern the methods used in and the facilities and controls used for the design, manufacture, packaging, labelling, storage, installation and servicing of all finished medical devices intended for human use", as described in the most recent revision.
Good Laboratory Practices (GLPs) were made final in 1979, and were defined as follows:

"... good laboratory practices for conducting nonclinical laboratory studies that support or are intended to support applications for research or marketing permits for products regulated by the Food and Drug Administration, including food and colour additives, animal food additives, human and animal drugs, medical devices for human use, biological products, and electronic products.

Similar GLP guidelines were adopted by the Organization for Economic Cooperation and Development (OECD) as shown below. The multilateral agreement is composed of three OECD Council Acts (adopted by OECD ambassadors):

i) The 1981 Council Decision on the Mutual Acceptance of Data in the Assessment of Chemicals (revised in 1997) that states that test data generated in any member country in accordance with OECD Test Guidelines and OECD Principles of Good Laboratory Practice (GLP) shall be accepted in other member countries for assessment purposes and other uses relating to the protection of human health and the environment.

ii) The 1989 Council DecisionRecommendation on Compliance with Principles of Good Laboratory Practices which establishes procedures for monitoring GLP compliance through government inspections and study audits as well as a framework for international liaison amongst monitoring and data-receiving authorities.

iii) The 1997 Council Decision on the Adherence of Non-Member countries to the Council Acts related to the Mutual Acceptance of Data in the Assessment of Chemicals that sets out a stepwise procedure for nonOECD economies to take part as full members in this system.

In the 1980s, the FDA began publishing a series of guidance documents that have had a major effect on our interpretation of current GMPs.

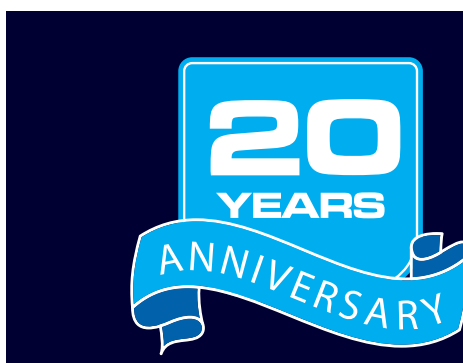

\section{The first company} worldwide to achieve ISO/IEC 17025

accreditation for liquid and glass CRMs

.and the preferred supplier

to leading pharmaceutical

companies, instrument

manufacturers and accredited laboratories globally.

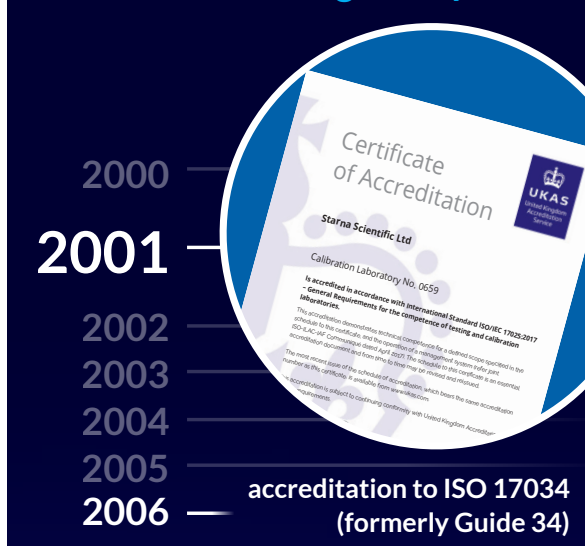

2007

2008

2009

2010

2011

2012

2013

2014

2015

2016

2017

2018

2019

2020
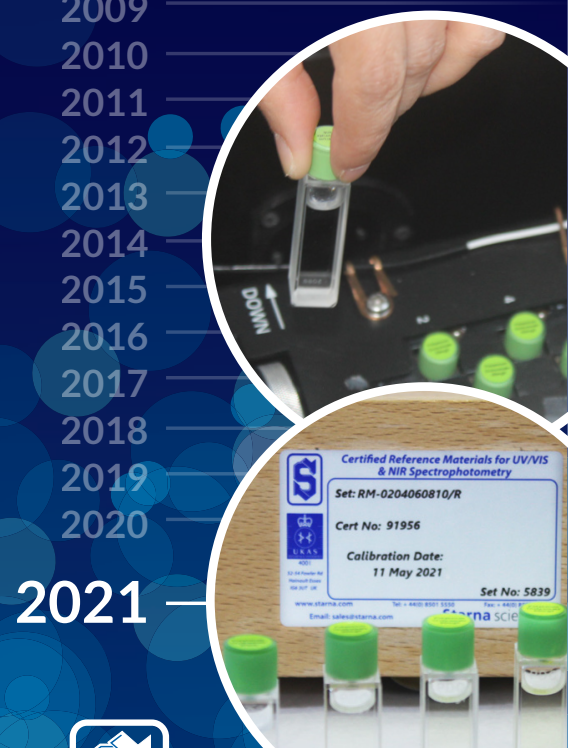

圆

Starna

Starna Scientific Ltd www.starna.com 
First, the Guide to Inspection of Computerized Systems in Drug Processing was published in 1983, which gave early expectations for the functioning of computer systems and perhaps signalled the beginning of computer validation.

Second, in 1987, the Guideline on General Principles of Process Validation outlined current thinking or expectations of process validation for drugs and devices.

Such documents, provide guidance only on principles and practices that are not legal requirements. However, as we have often discussed in this column, and will no doubt continue, ad infinitum, the line between what is a "standard" and what is purely "guidance" is sometimes a very thin tightrope indeed? Very often, as such, guidelines are published to reflect current regulatory agency thinking and expectations.

Last, a large percentage of the APIs used to manufacture products within a defined facility may come from sources outside the country, where manufacturing standards may not be as stringent. For this reason, at the end of this generation, both the European Union and the United States published draft guidance documents for the manufacture of APIs. The draft US document "Guidance for Industry: Manufacturing, Processing, or Holding of Active Pharmaceutical Ingredients" was released in 1998. Drug GMPs (21 CFR 210-211) are also considered to apply to the manufacture of APIs.

Also in the 1990s, proposed revisions to the GMPs for drugs and biologics were issued.

The Electronic Records Final Rule (21 CFR Part 11) was published in 1997 and required controls that ensure the security and accuracy of all data and computer systems used, i.e. software used in a GxP environment, must be CFR21 Part 11 compliant.

From a personal perspective, in the last five years of this period, I was involved with the development of UV/ Visible instrument systems, composing both hardware and software designed specifically to assist compliance with these new and challenging requirements, so saw the impact of these requirements at first hand. The interesting "switch" between the two tracks was that our organisation was certified to ISO 9001, which at that time also included specific clauses related to the design process, including software development; and which provided invaluable assistance in complying with these new GxP regulations.

The International Conference on Harmonization ( $\mathrm{ICH}$ ) was formed in 1990, and to this day is a consortium of international organisations ${ }^{2}$ working on a number of quality, safety and effectiveness documents. As those documents are adopted or made final by $\mathrm{ICH}$, they become "industry practice" in all participating countries. The 1996 ICH E6 guidance on good clinical practices has become the de facto standard on performing human clinical trials. A number of other guidance documents, including a draft guidance on handling out-of-specification results, were made available at this time. Even though these guidelines and draft guidances are not legally binding, they represent current thinking on their subject matter and tend to be adopted rapidly and/or viewed as "current industry practice."

\section{$3^{\text {rd }}$ Generation: the years 2000 to 2020}

Various keynote speeches by FDA insiders early in the 21 st century (in addition to high-profile audit findings focusing on computer system compliance) resulted in many companies scrambling to mount a defence against rule enforcement that they were procedurally and technologically unprepared for. Many software and instrumentation vendors released Part 11 "compliant" updates that were either incomplete or insufficient to fully comply with the rule. Complaints about the wasting of critical resources, non-valueadded aspects, in addition to confusion within the drug, medical device, biotech/ biologic and other industries about the true scope and enforcement aspects of Part 11 resulted in the FDA release of "FDA Guidance for Industry Part 11, Electronic Records: Electronic Signatures - Scope and Application (2003)".

This document was intended to clarify how Part 11 should be implemented and would be enforced. But, as with all FDA guidances, it was not intended to convey the full force of law-rather, it expressed the FDA's "current thinking" on Part 11 compliance. Many within the industry, while pleased with the more limited scope defined in the guidance, commented that, in some areas, the 2003 guidance contradicted requirements in the 1997 Final Rule.

In May 2007, the FDA issued the final version of their guidance on computerised systems in clinical investigations. This guidance supersedes the guidance of the same name dated April 1999; and supplements the guidance for industry on Part 11, Electronic Records; Electronic Signatures - Scope and Application and the Agency's international harmonisation efforts when applying these guidances to source data generated at clinical study sites.

Software requirements for Good Laboratory Practices (GLP), Good Manufacturing Practices (GMP) and Good Clinical Practice (GCP) require the following:

- An audit trail at the point in time when a record is first saved to durable media.

- The audit trail must contain the date and time stamp of the change, the description of the change, the reason for the change and the name of the person making that change.

- The audit trail must not obscure previous values-so both the old value and the new value for a given parameter must be recorded.

- Specific user accounts.

- Forward compatibility of all files generated by the software.

- All records, including audit trail records, must be protected from tampering.

CFR21 Part 11 compliance is necessary if results from the software are sent directly, electronically to the FDA or regulatory bodies as part of submissions.

Since June 2007, a different set of CGMP requirements have applied to all manufacturers of dietary supplements, with additional supporting guidance issued in 2010. Additionally, in the US, medical device manufacturers must follow what are called "quality system 
regulations" which are deliberately harmonised with ISO requirements, not necessarily CGMPs, and again we see another set of crossover points on the railway tracks.

In addition, and reviewed in previous articles, during this period, both the European and US Pharmacopoeias produced significant changes to reflect the changing environment, and some of the specific changes in the context of this discussion will be discussed in the next article.

However, it is worthy of note at this point that in 2005 the European Pharmacopoeia revised their UV/Vis General Chapter to allow the use of "... alternative Certified Reference Materials (CRMs)"-another "set of points" along the tracks, given that CRMs are defined by ISO/REMCO (now ISO TC 334).

During the 2005-2010 cycle, the USP also began a review of its instrument/system requirements described in General Chapter <851>, Spectrophotometry and Light Scattering. This chapter covered many spectroscopic techniques and had remained essentially unchanged over many years. Therefore, in the USP Review Cycle 2010-2015, it was decided to generate specific pairs of chapters for each of the main spectroscopic types. The chapters numbered below 1000 would define minimum standards for compliance for use in a monograph, the paired above 1000 chapter would give theory, guidance and recommendations for best analytical practices. In general, below 1000 chapters include procedures, instrument qualification and validation/verification sections. Each section involves an assessment of methodspecific requirements to ensure the suitability of the system and related measurements. General Chapter $<851>$, itself would be deleted after all the new chapters were approved.

The structure of this revision was discussed in an article from $2015,{ }^{3}$ with an update to the revisions in $2017 .{ }^{4}$

At the end of this period and in the 2015-2020 USP cycle we saw an increasing emphasis on "Data Integrity" and the introduction of a Lifecycle approach to all the key processes, both in manufacturing and the laboratory processes with the potential for future changes to GMP and GLP protocols?

\section{$4^{\text {th }}$ Generation: from 2021 forward}

In this current generation, as stated above, the initial forays into these key topics will be taken forward to proposed guidance on, for example, manufacturing, in addition to the continuing use of Process Analytical Technology (PAT), the control of continuous manufacturing processes and in the laboratory the application of the Lifecycle approach to the validation of the analytical measurement process. Both of which introduce new and somewhat related concepts and terminology.

In conclusion, referring and returning to our railway tracks analogy, we would again ask the question:

"Will their paths converge..."

And from a personal perspective, I would respond that maybe they will never converge completely, but at least, as this article has shown, unsurprisingly there are many similarities in the Quality requirements between GXP and the ISO 17xxx standards, which at first do not appear to be present-so there are some "points in the tracks".

And the last of this can be reflected in our $4^{\text {th }}$ generation comments. Associated with both GxP and ISO 17xxx is the management and minimisation of Risk, reflected by the adoption of a Lifecycle approach, i.e. it is not just a simple point-in-time validation, but an ongoing process. In the latest version of ISO/IEC 17025 , the evaluation of Risk is a specific requirement.

However, in both of these environments there appears to be an ongoing and continuous debate as to how these guidance documents and standards are implemented.

On the one hand, there is the position that these standards offer a framework to assist compliance, and it is up to the individual organisation to establish their own specific requirements within the guidelines.

On the other hand, there is the position that a standard should specifically state the requirements, and how these are to be achieved, and if these considerations are met then you are deemed to be "in compliance".

The next (and future) article(s) will enter into some of these more specific requirements, and again look to compare and contrast the above debate, in their implementation in the two key Quality areas, for example in "Pharmacopoeial compliance" and latest "ISO revisions".

"Will their paths converge... only time will tell?"

\section{References}

1. B.K. Immel, "A brief history of the GMPs for pharmaceuticals", Pharm. Technol. July (2001).

2. ICH Website: https://www.ich.org/ page/members-observers

3. C. Burgess and J.P. Hammond, "Modernisation of the spectroscopic General Chapters in the United States Pharmacopeia (USP)", Spectrosc. Europe 27(1), 26-29 (2015). https://doi.org/10.1255/sew.2015. a 1

4. C. Burgess and J.P. Hammond, "Update on the modernisation of the spectroscopic General Chapters in the United States Pharmacopeia (USP)", Spectrosc. Europe 29(6), 15-17 (2017). https://doi. org/10.1255/sew.2017.a2

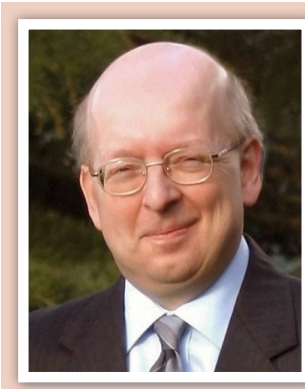

John Hammond is an experienced analytical scientist, spectroscopist and technical marketing professional, skilled in the development, production and marketing of analytical systems into highly regulated and controlled industries. A Fellow of the Royal Society of Chemistry (FRSC), executive member of ISO/TC334 and an Expert Advisor to the United States Pharmacopeia, General Chapters, Chemical Analysis committee. j.p.hammond@starna.com 